OPEN ACCESS

Edited by:

Ana Maria Cetto,

Universidad Nacional Autónoma de

México, Mexico

Reviewed by:

Luis De La Peña,

National Autonomous University of

Mexico, Mexico

Yilun Shang,

Northumbria University,

United Kingdom

Daniel C. Cole,

Boston University, United States

${ }^{*}$ Correspondence:

Theo M. Nieuwenhuizen

t.m.nieuwenhuizen@uva.n

Specialty section:

This article was submitted to Mathematical and Statistical Physics,

a section of the journal

Frontiers in Physics

Received: 09 March 2020

Accepted: 20 July 2020

Published: 23 October 2020

Citation:

Nieuwenhuizen TM (2020) Stochastic Electrodynamics: Renormalized Noise in the Hydrogen Ground-State Problem. Front. Phys. 8:335

doi: 10.3389/fphy.2020.00335

\section{Stochastic Electrodynamics: Renormalized Noise in the Hydrogen Ground-State Problem}

\author{
Theo M. Nieuwenhuizen* \\ Institute for Theoretical Physics, University of Amsterdam, Amsterdam, Netherlands
}

The hydrogen ground-state problem is a touchstone for the theory of Stochastic Electrodynamics. Recently, we have shown numerically and theoretically that the $\mathrm{H}$-atom self-ionizes after a characteristic time. In another approach, we reconsidered the harmonic oscillator and renormalized the stochastic force in order to suppress high-frequency tails so that all frequency integrals are dominated by the physical resonances. In the present work, we consider the regularization of the noise in the hydrogen ground-state problem. Several renormalization schemes are considered. Some are well-behaved, whereas in others the high frequency renormalization induces pathologies at low frequencies. In no situation did we find a way to escape from the previously signaled self-ionization.

Keywords: stochastic electrodynamics, hydrogen problem, hydrogen ground state, self-ionization, renormalization

\section{INTRODUCTION}

Stochastic electrodynamics (SED) is a classical theory that aims to explain quantum phenomena. Particles move in classical orbits. The basic assumption is the existence of a physical stochastic electromagnetic force that fills the universe and acts as an environment on charged particles and causes their quantum behavior at a statistical level. There is much literature on this field, and it can be summarized in the excellent books $[1,2]$.

The two celebrated touchstones of quantum physics, the harmonic oscillator [3-6] and the hydrogen problem [7-9], have received much attention within SED. The harmonic oscillator leads to a reasonable agreement, though not all details coincide. While the outcomes of various frequency integrals were routinely taken from their resonances, we have recently introduced a renormalization of the stochastic force such that high-frequency pathologies do not occur [10].

Our studies of the H-problem go back two decades. In [11], we showed how a classical phase space distribution can produce the shape of the quantum ground state, even Dirac's square-root shape, including relativistic corrections.

Stability of circular orbits was demonstrated by [12-14]. The numerics of the hydrogen ground state were performed in 2002 by [15] with a modestly optimistic outlook. With the aim to reconsider the problem, new simulations were performed in our group in 2016. Various schemes for treating the stochastic force numerically were formulated analytically. Liska employed video cards and a modern computer code, speeding up the simulations significantly. They were carried out for the non-relativistic problem [16] and with the inclusion of relativistic corrections [17]. Many CPU hours were spent to achieve long run times and to incorporate many frequency modes. Ongoing findings of self-ionization led to simulation of a variety of formulations of the problem. The bottom 
line was that there was always self-ionization, suggesting that SED is not a basis for quantum mechanics.

On another track, Huang and Batelaan [18] reported that quantum interferences do not show up in the SED version of a double-slit-like quantum model.

Nieuwenhuizen [19] showed analytically for the $\mathrm{H}$ atom that there is a trend for self-ionization when the energy of the elliptic orbit is close to zero and the dimensionless angular momentum lies below a critical value of order unity, thus supporting the numerics and the non-recurrence of orbits found by [8].

The question of whether a proper definition of SED can describe the hydrogen atom is of fundamental interest. It is the purpose of the present work to reinspect stability in the hydrogen ground-state problem, inspired by our recent renormalization of the stochastic force for the harmonic oscillator. In section 2, we recall some properties of elliptic orbits in the Kepler problem. In section 3, we consider energy absorption from the stochastic field for various renormalizations of the force. We close with a discussion.

\section{KEPLER ORBITS}

We consider an electron bound to a nucleus with charge $Z e$ and employ the notation of our recent work [19]. Lengths are expressed in terms of the Bohr radius $\hbar / \alpha Z m_{e} c$, times in the Bohr time $\hbar / \alpha^{2} Z^{2} m_{e} c^{2}$, speeds in the Bohr speed of $\alpha Z c$, energy in the Bohr energy $\alpha^{2} Z^{2} m_{e} c^{2}$, and angular momentum in terms of $\hbar$. Here, $\hbar$ is the reduced Planck constant, $\alpha \approx 1 / 137$ the fine structure constant, $Z$ the atomic number, $m_{e}$ the electron mass, and $c$ the speed of light.

We start recalling the essential details of the dynamics. In Bohr units the classical Newton equation reads

$$
\ddot{\mathbf{r}}=-\frac{\mathbf{r}}{r^{3}} .
$$

The Kepler orbit is solved in the parametric forms

$$
\begin{aligned}
\mathbf{r} & =\frac{1-\varepsilon \cos a}{k^{2}}(\cos \phi, \sin \phi, 0) \\
& =\frac{\left(c_{a}-\varepsilon, \kappa s_{a}, 0\right)}{k^{2}}
\end{aligned}
$$

Here, $\phi$ is the angle of the orbit with respect to the $x$-axis, $a$ is a time-like parameter, $\varepsilon$ is the ellipticity, and $\kappa=\sqrt{1-\varepsilon^{2}}$. Furthermore, $c_{a}$ is a shorthand for $\cos a$ and $s_{a}$ for $\sin a$. The orbit lies on the ellipse

$$
\left(k^{2} x+\varepsilon\right)^{2}+\frac{k^{4}}{\kappa^{2}} y^{2}=1
$$

Its perihelion lies at $\mathbf{r}=\mathbf{0}$, the location of the nucleus, and the aphelion at $\left(-2 \varepsilon / k^{2}, 0,0\right)$.

Time $t$ and a second time $s$ are parameterized as

$$
\begin{aligned}
& t=\frac{\tau_{a}}{k^{3}}, \quad \tau_{a}=a-\varepsilon \sin a, \\
& s=\frac{\tau_{b}}{k^{3}}, \quad \tau_{b}=b-\varepsilon \sin b .
\end{aligned}
$$

For circular orbits $(\varepsilon=0), \tau_{a}=a$ is a scaled time. In general, (2.4) exhibits an oscillation on top of this.

The angle $\phi$ is related to the variable $a$ as

$$
c_{\phi}=\frac{c_{a}-\varepsilon}{1-\varepsilon c_{a}}, \quad s_{\phi}=\frac{\kappa s_{a}}{1-\varepsilon c_{a}},
$$

and reads explicitly

$$
\phi=2 \arctan \left(\sqrt{\frac{1+\varepsilon}{1-\varepsilon}} \tan \frac{a}{2}\right) .
$$

It exhibits the ongoing revolutions; for circular orbits $(\varepsilon=0)$ it equals $\phi=a=k^{3} t$. For general $\varepsilon$, the orbit and $t$ are thus explicit in terms of $a$.

In Bohr units, the energy is $E=-\frac{1}{2} k^{2}$ and $\kappa=k L$ with $L$ being the angular momentum in units of $\hbar$. The period reads $P=2 \pi / k^{3}$. While the QM ground state corresponds to $k=1$, in SED, $k$ takes any value between 0 and $\infty$, that is, ranging from loosely to strongly bound, respectively. In the philosophy of SED, the time average of $E$ produces the ground state energy $E_{0}=-\frac{1}{2}$ as the average of $E$ over the stationary distribution of $E$-values. Presuming that it exists, its form has been determined in [11].

Linear perturbations $\mathbf{h}$ to the Kepler orbit satisfy

$$
\ddot{\mathbf{h}}(t)=-\mathbf{W}(t) \cdot \mathbf{h}(t), \quad \mathbf{W}=\frac{\mathbf{1}-3 \hat{\mathbf{r}} \hat{\mathbf{r}}}{r^{3}} .
$$

In [10], we presented a set of eigenmodes in the rotating frame. A linear combination of these solutions reads, in the laboratory frame,

$$
\begin{aligned}
\mathbf{h}^{(1)}(t) & =\frac{1}{\rho_{a}}\left(-s_{a}, \kappa c_{a}, 0\right), \\
\mathbf{h}^{(2)}(t) & =2\left(\varepsilon-c_{a},-\kappa s_{a}, 0\right)+3 \tau_{a} \mathbf{h}^{(1)}(t), \\
\mathbf{h}^{(3)}(t) & =\frac{1}{2 \rho_{a}}\left(-\kappa s_{2 a}, 3-4 \varepsilon c_{a}+c_{2 a}\right), \\
\mathbf{h}^{(4)}(t) & =\frac{\kappa}{2 \rho_{a}}\left(3-2 \varepsilon c_{a}-c_{2 a}, \frac{2 \varepsilon s_{a}-s_{2 a}}{\kappa}, 0\right), \\
\mathbf{h}^{(5)}(t) & =\left(0,0, s_{a}\right), \\
\mathbf{h}^{(6)}(t) & =\left(0,0, c_{a}-\varepsilon\right) .
\end{aligned}
$$

The benefit of these modes is that the limits $\varepsilon \rightarrow 0$ or $\kappa \rightarrow 0$ to be taken in each of them.

The Greens function satisfies

$$
\begin{aligned}
\ddot{\mathbf{G}}(t, s)+\mathbf{W}(t) \cdot \mathbf{G}(s, t) & =\mathbf{1} \delta(t-s), \\
\mathbf{G}^{\prime \prime}(t, s)+\mathbf{G}(t, s) \cdot \mathbf{W}(s) & =\mathbf{1} \delta(t-s),
\end{aligned}
$$

where dots denote derivatives to $t$ and primes to $s$. Generally, it holds that

$$
\dot{\mathbf{G}}\left(t, t^{-}\right)=-\mathbf{G}^{\prime}\left(t, t^{-}\right)=\mathbf{1}, \quad \dot{\mathbf{G}}^{\prime}\left(t, t^{-}\right)=\mathbf{0} .
$$


Following the approach of [19], we verify that for $s<t$, the Greens function reads explicitly

$$
\mathbf{G}(t, s)=\sum_{i=1,3,5} \frac{\mathbf{h}^{(i)}(t) \mathbf{h}^{(i+1)}(s)-\mathbf{h}^{(i+1)}(t) \mathbf{h}^{(i)}(s)}{k^{3}} .
$$

while causality imposes $\mathbf{G}=\mathbf{0}$ for $s \geq t$.

\section{STOCHASTIC ELECTRODYNAMICS}

In SED the Kepler orbit is perturbed by the stochastic electric field $\mathbf{E}$ and the damping $\mathbf{D}$,

$$
\ddot{\mathbf{r}}=-\frac{\mathbf{r}}{r^{3}}-\beta \mathbf{E}+\mathbf{D}
$$

The small parameter $\beta$ is related to the fine structure constant

$$
\beta=\sqrt{\frac{2}{3}} \alpha^{3 / 2} Z \approx \frac{Z}{1965} .
$$

with charge $Z=1$ for hydrogen. The damping $\mathbf{D}(t)$ has been analyzed in full detail in [10]; Here, the standard approximation $\mathbf{D}=\beta^{2} \dddot{\mathbf{r}}$ suffices. The stochastic field satisfies

$$
\mathbf{E}(t)=-\dot{\mathbf{A}}(t)=-\ddot{\mathbf{C}}(t) .
$$

It has zero average and correlation functions

$$
\begin{aligned}
& C_{E E}(t-s)=\langle\mathbf{E}(t) \mathbf{E}(s)\rangle=\Re \frac{6 \times \mathbf{1}}{\pi\left(t-s-i \tau_{c}\right)^{4}}, \\
& C_{A A}(t-s)=\langle\mathbf{A}(t) \mathbf{A}(s)\rangle=\Re \frac{-\mathbf{1}}{\pi\left(t-s-i \tau_{c}\right)^{2}}, \\
& C_{C E}(t-s)=\langle\mathbf{E}(t) \mathbf{C}(s)\rangle=\Re \frac{-\mathbf{1}}{\pi\left(t-s-i \tau_{c}\right)^{2}}, \\
& C_{C C}(t-s)=\langle\mathbf{C}(t) \mathbf{C}(s)\rangle \\
&=\frac{-\mathbf{1}}{\pi} \Re \log \omega_{c}\left(t-s-i \tau_{c}\right),
\end{aligned}
$$

where $\tau_{c}=\alpha^{2} Z^{2}$ is the Compton time $\hbar / m c$ in Bohr units and $\omega_{c} \sim \alpha^{3} \log 1 / \alpha$ is a low frequency cutoff. These correlators are large at $s=t$.

The energy radiation is well-understood. Per revolution there is an energy loss

$$
(\Delta E)_{r a d}=-\beta^{2} k^{5} \pi \frac{3-\kappa^{2}}{\kappa^{5}} .
$$

The theme of the present work is the average energy gained from the field. It occurs at the rate

$$
\left\langle\dot{E}_{\text {field }}\right\rangle=\beta^{2} \int_{s_{0}}^{t} \mathrm{~d} s\langle\mathbf{E}(t) \cdot \dot{\mathbf{G}}(t, s) \cdot \mathbf{E}(s)\rangle
$$

where we must take $s_{0} \rightarrow-\infty$. Integrated over a period $P=$ $2 \pi / k^{3}$, it brings

$$
\left\langle\Delta E_{\text {field }}\right\rangle=\beta^{2} \int_{-P / 2}^{P / 2} \mathrm{~d} t \int_{s_{0}}^{t} \mathrm{~d} s I_{1}(t, s)
$$

with

$$
I_{1}(t, s)=C_{E E}(t-s) \operatorname{tr} \dot{\mathbf{G}}(t, s),
$$

This expression has been studied in our previous work. The $s$ integral has potentially dangerous behavior at $s=t$ where $\dot{\mathbf{G}}=\mathbf{1}$ and $C_{E E}(0)$ is very large. But the shape (3.4) of $C_{E E}$ implies that this high frequency effect has a vanishing contribution. Just leaving it out corresponds to a motivated short-time $(t \approx s)$ or high frequency renormalization. The remaining integrand

$$
\dot{g}(t, s)=\operatorname{tr} \dot{\mathbf{G}}(t, s)-3=O\left[(t-s)^{4}\right],
$$

decays rapidly enough to set $\tau_{c} \rightarrow 0$ in $C_{E E}$ so that the integral is well behaved in this limit.

\subsection{Short-Time Regularization}

In our recent study of the harmonic oscillator we introduced a high-frequency regularization of the ultraviolet contributions [10]. Leaving out the subleading damping $\mathbf{D}$, it amounts to replace $\mathbf{E} \rightarrow \overline{\mathbf{E}}$, where the frequency components are related as

$$
\overline{\mathbf{E}}_{\omega} \equiv \frac{\omega_{0}^{2}}{\omega^{2}} \mathbf{E}_{\omega}=\omega_{0}^{2} \mathbf{C}_{\omega}
$$

with the equality from $\mathbf{E}(t)=-\ddot{\mathbf{C}}(t)$. At the resonance frequency $\omega=\omega_{0}$, the $\overline{\mathbf{E}}_{\omega}$ and $\mathbf{E}_{\omega}$ coincide. For nonlinear potentials this demands a generalization. The definition of $\mathbf{G}$ is $\ddot{\mathbf{G}}+\mathbf{W} \cdot \mathbf{G}=$ $\mathbf{1} \delta(t-s)$ in the hydrogen problem, while $\mathbf{W}(t) \rightarrow \mathbf{1} \omega_{0}^{2}$ in the harmonic case. A natural and simple generalization is therefore

$$
\overline{\mathbf{E}}(t)=\mathbf{W}(t) \cdot \mathbf{C}(t) .
$$

Indeed, this reduces to (3.10) for the harmonic case. With $\overline{\mathbf{E}}$ instead of $\mathbf{E}$ inserted in (3.1), there will now appear in (3.7) the renormalized integrand

$$
\begin{aligned}
I_{2} & =\langle\overline{\mathbf{E}}(t) \cdot \dot{\mathbf{G}}(t, s) \cdot \overline{\mathbf{E}}(s)\rangle \\
& =C_{C C}(t-s) \operatorname{tr} \mathbf{W}(t) \cdot \dot{\mathbf{G}}(t, s) \cdot \mathbf{W}(s) .
\end{aligned}
$$

We also consider the expressions with one $\mathbf{E}$ and one $\overline{\mathbf{E}}$, which result in

$$
\begin{aligned}
& I_{3}=C_{C E}(t-s) \operatorname{tr} \dot{\mathbf{G}}(t, s) \cdot \mathbf{W}(s), \\
& I_{4}=C_{C E}(t-s) \operatorname{tr} \mathbf{W}(t) \cdot \dot{\mathbf{G}}(t, s) .
\end{aligned}
$$

By partial integration we can generally relate the $s$-integral over $I_{1}$ to one over $I_{4}$.

$$
\begin{aligned}
& \int_{-\infty}^{t} \mathrm{~d} s \dot{\mathbf{G}}(t, s) \Re \frac{6}{\pi\left(t-s+i \tau_{c}\right)^{4}}= \\
& \int_{-\infty}^{t} \mathrm{~d} s \dot{\mathbf{G}}(t, s) \mathbf{W}(s) \Re \frac{-1}{\pi\left(t-s+i \tau_{c}\right)^{2}} .
\end{aligned}
$$


In the boundary terms, we used $\dot{\mathbf{G}}^{\prime}(t, t)=\mathbf{0}$ and inserted $\dot{\mathbf{G}}^{\prime \prime}(t, s)=-\dot{\mathbf{G}}(t, s) \cdot \mathbf{W}(s)$. But when we do the same to relate $I_{3}$ to $I_{2}$, we cannot omit the boundary terms at large negative $s_{0}$,

$$
\begin{aligned}
& \int_{s_{0}}^{t} \mathrm{~d} s \Re \frac{-\dot{\mathbf{G}}(t, s)}{\pi\left(t-s+i \tau_{c}\right)^{2}}=\frac{\dot{\mathbf{G}}\left(t, s_{0}\right)}{\pi\left(t-s_{0}\right)} \\
& +\dot{\mathbf{G}}^{\prime}\left(t, s_{0}\right) \frac{\log \omega_{1}\left(t-s_{0}\right)}{\pi} \\
& -\int_{s_{0}}^{t} \mathrm{~d} s \dot{\mathbf{G}}(t, s) \cdot \mathbf{W}(s) \frac{\log \omega_{1}(t-s)}{\pi}
\end{aligned}
$$

where we took $\tau_{c} \rightarrow 0$ in the right-hand side. The main reason for the complication is that $\mathbf{G}(t, s)$, as well as its derivatives, contain an explicit factor $t-s$ arising from the secular part $3 k^{3} t \mathbf{h}^{(1)}(t)$ of the $\mathbf{h}^{(2)}(t)$ mode, see (2.8). With the left-hand side of (3.15) well-behaved for $s_{0} \rightarrow-\infty$, it follows that the integral in the right hand side must have an $s_{0}+s_{0} \log \left|s_{0}\right|$ divergency in this limit. This is confirmed by inspection and implies that the short-time regularization (2.8) creates a longtime divergency. It is related to the $1 / \omega^{2}$ factor in (3.10) and already led for the harmonic oscillator to a divergency; this was, however, subdominant. For the hydrogen problem it is more cumbersome and leads to an ill-defined leading order integral over $I_{2}$. Similar computational methods of integral calculation have been used in other settings (see e.g., [20]).

Though $C_{C C}$ in Equation (3.4) involves a cutoff $\omega_{c}$, Equation (3.15) is valid for any $\omega_{1}$. But even the awkward choice $\omega_{1} \sim$ $-1 / s_{0}$ would not eliminate the boundary terms that regularize the integral.

\subsection{Nearing the Self-Ionization}

The important question of whether the $\mathrm{H}$ ground state is stable in SED is analyzed for orbits in the limit where $E=-\frac{1}{2} k^{2}$ vanishes. In our previous works, we showed that this amounts to studying the orbits in the limit where $\kappa=k L$ vanishes, at fixed $L$, in an order unity. From (3.5), one has the energy loss by radiation per orbit

$$
(\Delta E)_{\text {rad }} \approx-3 \pi \frac{\beta^{2}}{L^{5}} .
$$

To study this limit, the scaling $a \rightarrow \kappa u, b \rightarrow \kappa v$ for $\kappa \rightarrow 0$ is introduced, expressing that the main contribution, described by $u$ and $v$ of the order unity, comes from the part of the Kepler orbit near the pericenter at $u=0$. Indeed, it holds that

$$
\mathbf{r}=\frac{L^{2}}{2}\left(1-u^{2}, 2 u, 0\right), r=\frac{L^{2}}{2}\left(1+u^{2}\right) .
$$

Clearly, this part of the orbit is in its $k \rightarrow 0$ limit, while the farthest point, lying at $\left((1+\varepsilon) / k^{2}, 0,0\right) \approx\left(2 / k^{2}, 0,0\right)$, exhibits a self-ionization for $k \rightarrow 0$. For further details of the method we refer to [19]. We reproduce its equations (2.24)-(2.26) for $\kappa \rightarrow 0$ and multiplied by $P$,

$$
\begin{aligned}
& \left\langle\Delta E_{\text {field }}^{(1)}\right\rangle=\frac{144}{5 \pi} \frac{\beta^{2}}{L^{6}} \int_{-\infty}^{\infty} \mathrm{d} u \int_{-\infty}^{u} \mathrm{~d} v \times \\
& \frac{27}{2} \frac{5+3 u^{2}+4\left(2+u^{2}\right) u v+\left(u^{2}-1\right) v^{2}}{\left(1+u^{2}\right)^{2}\left(3+u^{2}+u v+v^{2}\right)^{4}} .
\end{aligned}
$$

Continuing along these lines, we find that $\left\langle\Delta E_{\text {field }}^{(3)}\right\rangle$ is equal to this, while $\left\langle\Delta E_{\text {field }}^{(4)}\right\rangle$ comes out with the second line replaced by

$$
\begin{aligned}
& \frac{15+20 u^{2}+3 u^{4}+4\left(5+8 u^{2}+u^{4}\right) u v}{\left(1+u^{2}\right)^{5}\left(3+u^{2}+u v+v^{2}\right)^{2}}+ \\
& \frac{5+u^{2}+8 u^{4}+2\left(5+u^{2}\right) u v+\left(u^{2}-1\right) v^{2}}{\left(1+u^{2}\right)^{5}\left(3+u^{2}+u v+v^{2}\right)^{2}} v^{2} .
\end{aligned}
$$

Its $v$-integral is linearly divergent with logarithms, as it is for $\left\langle\Delta E_{\text {field }}^{(2)}\right\rangle$. This all results in

$$
\begin{aligned}
\left\langle\Delta E_{\text {field }}^{(1)}\right\rangle & =\frac{16 \sqrt{3}}{5} \frac{\beta^{2}}{L^{6}} \\
\left\langle\Delta E_{\text {field }}^{(2)}\right\rangle & =\text { divergent } \\
\left\langle\Delta E_{\text {field }}^{(3)}\right\rangle & =\frac{16 \sqrt{3}}{5} \frac{\beta^{2}}{L^{6}} \\
\left\langle\Delta E_{\text {field }}^{(4)}\right\rangle & =\text { divergent }
\end{aligned}
$$

The equality of the first and third case yields some justification for the renormalization method we investigated.

In case 1 and 3, the average total energy change per orbit thus comes out as

$$
\begin{aligned}
\Delta E & =3 \pi \frac{\beta^{2}}{L^{6}}\left(L_{c}-L\right), \\
L_{c} & =\frac{16}{5 \pi \sqrt{3}}=0.588057 .
\end{aligned}
$$

Orbits that have achieved a small $k$ and $L<L_{c}$ will gain energy on average, which explains the self-ionization observed in all our numerics.

\subsection{Other Renormalization Schemes}

The renormalization $\mathbf{E} \rightarrow \overline{\mathbf{E}}=\mathbf{W}(t) \cdot \mathbf{C}(t)$ involves $\mathbf{W}=$ $(\mathbf{1}-3 \hat{\mathbf{r}} \hat{\mathbf{r}}) / r^{3}$, of which the numerator has eigenvalues -2 and 1 (twice). One may wonder whether the "absolute value" $|\mathbf{W}| \equiv$ $(\mathbf{1}+\hat{\mathbf{r}} \hat{\mathbf{r}}) / r^{3}$, with the eigenvalues +2 and 1 (twice), fares better. Inspection shows that the divergence does not disappear; if anything, it becomes worse.

A renormalization with a broken power of $|\mathbf{W}|$ fares better at large times. One may replace $\mathbf{E}=-\dot{\mathbf{A}}$ by $\overline{\mathbf{E}}(t)=-\sqrt{|\mathbf{W}|(t)} \cdot \mathbf{A}(t)$ with the expression $\sqrt{|\mathbf{W}|}=(\mathbf{1}+(\sqrt{2}-1) \hat{\mathbf{r}} \hat{\mathbf{r}}) / r^{3 / 2}$ squaring to $|\mathbf{W}|$. Like (3.11), this approach softens the short time behavior, but it does not ruin the long time regime. This leads to a contribution to $\langle\dot{E}\rangle_{\text {field }}$ of the form

$$
\begin{aligned}
& \int_{-\infty}^{t} \mathrm{~d} s \Re \frac{-f(t, s)}{\left(t-s+i \tau_{c}\right)^{2}}=f^{\prime}(t, t)\left|\log \tau_{c}\right| \\
+ & \int_{-\infty}^{t} \mathrm{~d} s f^{\prime \prime}(t, s) \log (t-s)+O\left(\tau_{c}\right) .
\end{aligned}
$$

Using $\dot{\mathbf{G}}=\mathbf{1}$ and $\dot{\mathbf{G}}^{\prime}=\mathbf{0}$ at $s=t$, the boundary term leads to

$$
\frac{\delta\langle\dot{E}\rangle_{\text {field }}}{\left|\log \tau_{c}\right|}=\frac{\beta^{2}}{2 \pi} \frac{\mathrm{d}}{\mathrm{d} t} \operatorname{tr}|\mathbf{W}|=-\frac{6 \beta^{2}}{2 \pi} \frac{\dot{r}}{r^{4}} .
$$


It expresses energy gain (i.e., the electron becomes less bound, on the average) on the approach to the pericenter, and loss (becoming more bound) on departure. This cutoff dependence is unexpected. Nevertheless, when integrated over a full period, the effect averages out.

Next, we calculate, in analogy with (3.7), the energy gain per period. In the scaling limit, the $t, s$ integrals become $u, v$ integrals, of which the latter can be performed analytically. Its $v=u$ boundary term vanishes upon $u$-integration, while the integral over the $v=-\infty$ boundary term leads to a finite result,

$$
\left\langle\Delta E_{\text {field }}\right\rangle=2.99842\left\langle\Delta E_{\text {field }}^{(1)}\right\rangle .
$$

Hence it also leads to self-ionization.

The combination $\overline{\mathbf{E}}=(1-x) \mathbf{E}-x \sqrt{|\mathbf{W}|} \cdot \mathbf{A}$ involves from the $\mathrm{AE}$ and $\mathbf{E A}$ cross terms, a new contribution of the form

$$
\begin{aligned}
& \int_{-\infty}^{t} \mathrm{~d} s \Re \frac{-2 f(t, s)}{\left(t-s+i \tau_{c}\right)^{3}}=f^{\prime \prime}(t, t) \log \tau_{c} \\
+ & \int_{-\infty}^{t} \mathrm{~d} s f^{\prime \prime \prime}(t, s) \log (t-s)+O\left(\tau_{c}\right) .
\end{aligned}
$$

with a lengthy $f$ having $f(t, t)=0$. The $\log \tau_{c}$ again drops out when integrated over a full period. After scaling, the $v$-integral can be performed analytically; now, the primitive for $v \rightarrow-\infty$ is odd in $u$, while the result comes from the $v=u$ term. This ends up in

$$
\begin{aligned}
& \left\langle\Delta E_{\text {field }}^{(x)}\right\rangle=\frac{16 \sqrt{3}}{5} \frac{\beta^{2}}{L^{6}} \times \\
& {\left[(1-x)^{2}-0.876444(1-x) x+2.99842 x^{2}\right] .}
\end{aligned}
$$

Its minimum at $x=0.295028$ leads to $L_{c}^{\min }=0.33855$, smaller than $L_{c}=0.58808$ from (3.21). For all $x$, this still leads to self-ionization.

The above "absolute" value $|\mathbf{W}|$ looks unnatural, but it was necessary to define a real valued version of $\sqrt{\mathbf{W}}$. The third roots are real however:

$$
\begin{aligned}
& \mathbf{W}^{1 / 3}=\left(\mathbf{1}-\left(2^{1 / 3}+1\right) \hat{\mathbf{r}} \hat{\mathbf{r}}\right) / r \\
& \mathbf{W}^{2 / 3}=\left(\mathbf{1}+\left(2^{2 / 3}-1\right) \hat{\mathbf{r}} \hat{\mathbf{r}}\right) / r^{2}
\end{aligned}
$$

It is easily verified that $\left(\mathbf{W}^{1 / 3}\right)^{2}=\mathbf{W}^{2 / 3}$ and $\left(\mathbf{W}^{1 / 3}\right)^{3}=\mathbf{W}$. They thus permit the renormalization

$$
\mathbf{E} \rightarrow \overline{\mathbf{E}}=(1-x) \mathbf{W}^{1 / 3} \cdot \mathbf{B}_{1}+x \mathbf{W}^{2 / 3} \cdot \mathbf{B}_{2},
$$

for some real valued $x$, with the stochastic fields

$$
\mathbf{B}_{1}=\partial_{t}^{-2 / 3} \mathbf{E}, \quad \mathbf{B}_{2}=\partial_{t}^{-4 / 3} \mathbf{E},
$$

defined by having $e^{-i \omega t}$ frequency components

$$
\left(\mathbf{B}_{1}\right)_{\omega}=\frac{\mathbf{E}_{\omega}}{(-i \omega)^{2 / 3}}, \quad\left(\mathbf{B}_{2}\right)_{\omega}=\frac{\mathbf{E}_{\omega}}{(-i \omega)^{4 / 3}} .
$$

In the notation of [10], their correlation functions $\left\langle\mathbf{B}_{i}(t) \mathbf{B}_{j}(s)\right\rangle=$ $\mathbf{1} B_{i j}(t-s)$ emerge as

$$
\begin{aligned}
B_{11}(t) & =\int_{-\infty}^{\infty} \frac{\mathrm{d} \omega}{2 \pi} \frac{|\omega|^{3}}{|\omega|^{4 / 3}} e^{-i \omega t-|\omega| \tau_{c}} \\
& =\frac{1}{\pi} \Gamma_{8 / 3} \Re \frac{1}{\left(i t+\tau_{c}\right)^{8 / 3}}, \\
B_{22}(t) & =\frac{1}{\pi} \Gamma_{4 / 3} \Re \frac{1}{\left(i t+\tau_{c}\right)^{4 / 3}}, \\
B_{12}(t) & =B_{21}(-t)=\frac{1}{\pi} \Re \frac{e^{-\pi i / 3}}{\left(i t+\tau_{c}\right)^{2}} .
\end{aligned}
$$

The difficulty is again to deal with the singularities in the limit $\tau_{c} \rightarrow 0$. To proceed, we perform partial integrations. We introduce $B_{11}^{(3)}$ and $B_{22}^{(1)}$ to get

$$
\begin{aligned}
& B_{11}=\dddot{B}_{11}^{(3)}, \quad B_{11}^{(3)}(t)=-\frac{27}{20 \pi} \Gamma_{8 / 3} t^{1 / 3}, \\
& B_{22}=\dot{B}_{22}^{(1)}, \quad B_{22}^{(1)}(t)=\frac{3}{2 \pi} \Gamma_{4 / 3} t^{-1 / 3}, \\
& B_{12}(t)=B_{21}(t)=-\frac{1}{2 \pi t^{2}},
\end{aligned}
$$

where we took $\tau_{c} \rightarrow 0$. In view of (3.21) we define

$$
\begin{aligned}
& L_{c}^{i j}=\frac{L^{6}}{3 \pi} \int_{-P / 2}^{P / 2} \mathrm{~d} t \int_{s_{0}}^{t} \mathrm{~d} s B_{i j}(t-s) \Gamma_{i j}(t, s), \\
& \Gamma_{i j}(t, s)=\operatorname{tr} \mathbf{W}^{i / 3}(t) \cdot \dot{\mathbf{G}}(t, s) \cdot \mathbf{W}^{j / 3}(s),
\end{aligned}
$$

for $i, j=1,2$. For $L_{c}^{11}$ we perform a partial integration w.r.t. $s$. Next we write the $t$-integral as the difference between two integrals starting at $s_{0}$ and switch the $t$ and $s$ integrals. Then we do a partial integration w.r.t. $t$, switch back and do a final one w.r.t. $s$. This leads to a $t, s$ integral over $-\dot{\Gamma}_{11}^{\prime \prime} B_{11}^{(3)}$. One boundary term at $t=s$ is non-trivial, namely

$$
\begin{aligned}
\delta L_{c}^{11} & =\frac{3 \Gamma_{8 / 3} L^{6}}{10 \pi^{2} \tau_{c}^{2 / 3}} \int_{-P / 2}^{P / 2} \mathrm{~d} s \Gamma_{11}^{\prime}(s, s) \\
& =-\frac{3\left(1-2^{-1 / 3}\right) \Gamma_{8 / 3} L^{6}}{5 \pi^{2} \tau_{c}^{2 / 3}} \int_{-P / 2}^{P / 2} \mathrm{~d} s \frac{r^{\prime}(s)}{r(s)^{3}} .
\end{aligned}
$$

This vanishes again since it involves a total derivative integrated over a full period. But the integrand itself is moderately large, so that, as before, the average rate of energy exchange with the field results in gain on approach to the pericenter and loss on departure. While weakened by the prefactor and canceling over a period, this cutoff dependence is unexpected.

For $L_{c}^{22}$ we perform a partial integration w.r.t. $s$ and evaluate the double integral in the limit $\tau_{c} \rightarrow 0$. The boundary term at $s=t$ vanishes identically. With $\Gamma^{12} \sim \Gamma^{21} \sim(t-s)^{2}$ for $s \rightarrow t$, the $L_{c}^{12}$ and $L_{c}^{21}$ integrands are already regular for $\tau_{c} \rightarrow 0$. 
We are interested in these results in the scaling limit $\kappa=$ $k L \rightarrow 0$ at fixed $L$. The resulting integrals are of the type (3.18). Numerical evaluation yields

$$
\begin{array}{ll}
L_{c}^{11}=8.5191, & L_{c}^{22}=2.1944 \\
L_{c}^{12}=0.3182, & L_{c}^{21}=-0.5615
\end{array}
$$

The combined $L_{c}$ corresponding to (3.28) reads

$$
L_{c}^{11}(1-x)^{2}+\left(L_{c}^{12}+L_{c}^{21}\right) x(1-x)+L_{c}^{22} x^{2} .
$$

It has a minimum at $x=0.7886$,

$$
L_{c}^{\min }=1.7048
$$

which sets the boundary for self-ionizing orbits because (3.36) exceeds this for other $x$-values.

\section{DISCUSSION}

Previous studies, both analytical and numerical, have pointed out that the hydrogen problem in Stochastic Electrodynamics leads to a self-ionization of the electron. The present work investigates whether "easy fixes" of the stochastic force may improve the situation. We consider a short time or high frequency renormalization of the stochastic force that we recently proposed for the harmonic oscillator problem and generalized it for the hydrogen ground-state problem. To achieve this, we consider several options, of which some do, and some do not, lead to a well-defined approach. We find that the renormalization does not help to stabilize the situation, and

\section{REFERENCES}

1. Cetto A, de la Peña L. The quantum dice, an introduction to stochastic electrodynamics. In: Fundamental Theories of Physics. Vol. 75 (1996). p. 1-512. doi: 10.1007/978-94-015-8723-5

2. de la Peña L, Cetto AM, Vald'es-Hern'andez A. The Emerging Quantum: The Physics Behind Quantum Mechanics. Berlin: Springer (2014).

3. Surdin M. Derivation of Schrödinger's equation from stochastic electrodynamics. IJTP. (1971) 4:117-23. doi: 10.1007/BF00670387

4. Santos E. The harmonic oscillator in stochastic electrodynamics. Il Nuovo Cimento B (1971-1996) (1974) 19:57-89. doi: 10.1007/BF027 49757

5. De la Peña L, Cetto A. The quantum harmonic oscillator revisited: a new look from stochastic electrodynamics. J Math Phys. (1979) 20:469-83. doi: 10.1063/1.524098

6. Boyer TH. A brief survey of stochastic electrodynamics. In: Foundations of Radiation Theory and Quantum Electrodynamics. Boston, MA: Springer (1980). p. 49-63. doi: 10.1007/978-1-4757-0671-0_5

7. Claverie P, Pesquera L, Soto F. Existence of a constant stationary solution for the hydrogen atom problem in stochastic electrodynamics. Phys Lett A. (1980) 80:113-6. doi: 10.1016/0375-9601(80)90198-X

8. Claverie P, Soto F. Nonrecurrence of the stochastic process for the hydrogen atom problem in stochastic electrodynamics. J Math Phys. (1982) 23:753-9. doi: $10.1063 / 1.525431$ that its impact on long time behavior actually makes the situation worse.

Next, we study various further renormalization schemes which lead to well behaved dynamics, but neither heal the selfionization problem. Our approach generally puts forward that stability of orbits with energy near $E=0$ can only be achieved for a scheme in which the parameter $L_{c}$ in (3.21) vanishes. On physical grounds one expects that it can be proven that this quantity is positive. However, we are not aware of such a proof, not even in the scaling limit $E \rightarrow 0$.

In our view, the problem does not lie in the Kepler orbits but in the close enough approach to the nucleus where a relatively high amount of energy is absorbed from the stochastic force. Indeed, Kepler orbits can be stable in SED. Nieuwenhuizen [19] adds an $L_{0}^{2} / 2 r^{2}$ potential to the $-1 / r$ Newton potential. It induces an effective angular momentum $L_{\text {eff }}=\left(L^{2}+L_{0}^{2}\right)^{1 / 2}$, which, if $L_{0} \gtrsim 6$ is large enough, leads to a stable system without self-ionization. Then $L_{e f f}$, and with it the distance between the pericenter and the nucleus, is large enough to prevent orbits that keep on gaining energy on the average.

In the absence of such an extra potential, we confirm previous findings that the hydrogen self-ionizes in Stochastic Electrodynamics. When the orbit has nearly zero energy and the angular momentum lies below some critical value, then, on the average, more energy gets absorbed from the field than is radiated away, making the orbit more and more delocalized so that ultimately self-ionization occurs. To circumvent this, a fundamental reformulation of Stochastic Electrodynamics seems to be necessary.

\section{AUTHOR CONTRIBUTIONS}

The author confirms being the sole contributor of this work and has approved it for publication.

9. França H, Franco H, Malta, C. A stochastic electrodynamics interpretation of spontaneous transitions in the hydrogen atom. Eur J Phys. (1997) 18:343. doi: 10.1088/0143-0807/18/5/006

10. Nieuwenhuizen TM. Stochastic electrodynamics: lessons from regularizing the harmonic oscillator. Atoms. (2019) 7:59. doi: 10.3390/atoms70 20059

11. Nieuwenhuizen TM. Classical phase space density for the relativistic hydrogen atom. AIP Conf Proc. (2006) 810:198. doi: 10.1063/1.21 58722

12. de la Peña L. Introducción a la Mecánica Cuántica. Mexico City: UNAM-FCE (1980).

13. Puthoff HE. Ground state of hydrogen as a zero-point-fluctuation-determined state. Phys Rev D. (1987) 35:3266. doi: 10.1103/PhysRevD.35.3266

14. Puthoff HE. Quantum ground states as equilibrium particle-vacuum interaction states. Quant Stud. (2012) 1-6.

15. Cole DC, Zou Y. Quantum mechanical ground state of hydrogen obtained from classical electrodynamics. Phys Lett A (2003) 317:14-20. doi: $10.1016 /$ j.physleta.2003.08.022

16. Nieuwenhuizen TM, Liska MT. Simulation of the hydrogen ground state in stochastic electrodynamics. Phys Scripta 2015) 2015:014006. doi: 10.1088/0031-8949/2015/T165/0 14006

17. Nieuwenhuizen TM, Liska MT. Simulation of the hydrogen ground state in stochastic electrodynamics-2: inclusion of relativistic 
corrections. Found Phys. (2015) 45:1190-202. doi: 10.1007/s10701-0159919-0

18. Huang WCW, Batelaan H. Testing quantum coherence in stochastic electrodynamics with squeezed schrödinger cat states. Atoms. (2019) 7:42. doi: 10.3390/atoms7020042

19. Nieuwenhuizen TM. On the stability of classical orbits of the hydrogen ground state in Stochastic Electrodynamics. Entropy. (2016) 18:135. doi: $10.3390 / \mathrm{e} 18040135$

20. Shang Y. The limit behavior of a stochastic logistic model with individual time-dependent rates. J Math. (2013) 2013:502635. doi: 10.1155/2013/502635
Conflict of Interest: The author declares that the research was conducted in the absence of any commercial or financial relationships that could be construed as a potential conflict of interest.

Copyright (c) 2020 Nieuwenhuizen. This is an open-access article distributed under the terms of the Creative Commons Attribution License (CC BY). The use, distribution or reproduction in other forums is permitted, provided the original author(s) and the copyright owner(s) are credited and that the original publication in this journal is cited, in accordance with accepted academic practice. No use, distribution or reproduction is permitted which does not comply with these terms. 